\title{
Anomalous origin of the coronary arteries-review article
}

Abdelrahman Aljadi, MD, MSc ${ }^{1}$, Majd Kanbour, MD ${ }^{1}$, Shaheed Elhamdani, MSIV ${ }^{1}$, Mehiar ElHamdani, MD, FACC, FSCAI ${ }^{1}$

\section{Author Affiliations:}

1. Marshall University Joan C. Edwards School of Medicine, Huntington, West Virginia

The authors have no financial disclosures to declare and no conflicts of interest to report.

\section{Corresponding Author}

Abdelrahman Aljadi, MD, MSc

Marshall University Joan C. Edwards School of Medicine

Huntington, West Virginia

Email: aljadi@marshall.edu 


\section{Abstract}

Anomalous origin of the coronary arteries is rare in clinical practice. Different anomalies have different clinical or symptomatic manifestations depending on the patient's age. However, the majority of coronary artery origin anomalies are silent and are only discovered incidentally during left heart catheterization (LHC). Acute myocardial infarction (MI) or even sudden death may occur in patients with an anomalous origin of the left main (LM) coronary artery from the right coronary cusp with a subsequent course between the aorta and the pulmonary artery. Therefore, recognition of anomalous origin of the coronary arteries is essential for clinical practice to further classify and properly manage patients with increased risk of acute coronary syndrome (ACS). Furthermore, patients found to have an anomaly may be eligible for surgical correction.

\section{Keywords}

Coronary artery, Anomalous origin, Left Main coronary artery (LM), Left Circumflex (LCX) artery, Right Coronary Artery (RCA), Right Coronary Cusp, Left Coronary Cusp, Acute Coronary Syndrome (ACS)

\section{Introduction}

The reported incidence of the anomalous origin of the coronary arteries is $0.28 \%$ to $1.3 \%$ of the population. ${ }^{1,2}$ Within this minority, the left circumflex artery originating from the right sinus of Valsava is the most common anomaly, with incidence of $0.4 \%{ }^{3}$ The right coronary artery arising from the left sinus of Valsalva or the left main coronary artery arising from the right sinus of Valsalva is less common with a prevalence in the population between $0.1 \%$ and $0.3 \%$. ${ }^{4}$ The prevalence of the RCA arising from the left sinus of Valsalva is greater than the left coronary artery from the right sinus of Valsalva. The prevalence of left main coronary artery originating from the right sinus of Valsalva is very rare and has been reported to be only $0.047 \% .^{5}$

The clinical presentation of the anomalous origin of the coronary arteries varies greatly based on different types of anomalies. Most patients are asymptomatic, but some may present with anginal chest pain or syncope with exertion. While anomalous origin of the left main coronary artery is the rarest, its clinical implications are the most serious, especially when the artery runs between the aorta and the pulmonary artery potentially leading to compression of the coronary artery and a subsequent acute myocardial infarction (MI) or sudden death. In a previous study by Maron BJ, et al, ${ }^{6}$ it was reported that coronary artery origin anomalies are second in frequency to hypertrophic cardiomyopathy as a cause of sudden death in young athletes. The incidence of sudden death in patients with anomalous origin of the left coronary artery is higher than in patients with anomalous origin of the right coronary artery especially in the absence of preceding symptoms. ${ }^{7}$ Due to the increased risk of cardiac ischemia, acute coronary syndrome (ACS), and sudden death in these patients, more aggressive modification of the modifiable risk factor of coronary artery diseases (CAD) and surgical correction of the coronary artery anomalies to prevent possible complications may be necessary. 


\section{Case presentation}

An 89 year old Caucasian female, with medical history significant for hypertension and no previous reported history of coronary artery disease (CAD), presented with dyspnea on exertion and worsening bilateral lower extremity edema. Social history was negative for any smoking or alcohol intake. Vital signs were stable. Cardiac examination showed regular rhythm and controlled rate without murmurs, gallops, or rubs. Chest $\mathrm{X}$ ray showed no evidence of pulmonary edema. EKG showed new $\mathrm{T}$ wave inversion in inferior and lateral chest leads. Troponin was mildly elevated. Echocardiogram showed normal Ejection Fraction (EF) with no wall motion abnormalities, dilated right ventricle with volume and pressure overload. Pulmonary embolism was excluded with CT chest. LHC was performed, and we found anomalous origin of the Left Main (LM) coronary artery. The

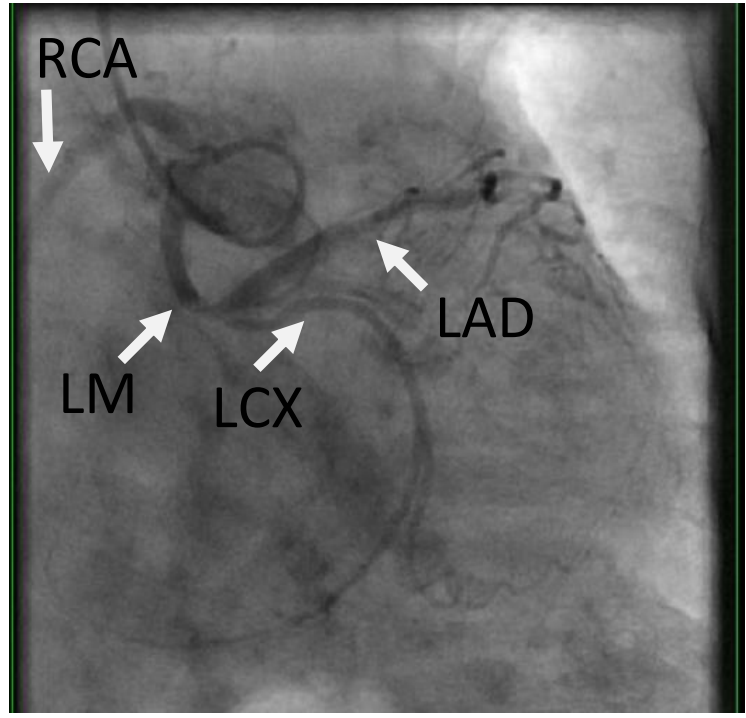

Figure 1. Left heart catheterization showing anomalous origin of left main from the right cusp.

LM coronary artery originated from the right coronary cusp and bifurcated into left anterior descending (LAD) artery and left circumflex (LCX) artery as shown in Figure 1. It was determined that she had had non-obstructive CAD, and optimal medical management was recommended.

\section{Variations of the anomalous origin of the coronary arteries}

Coronary artery origin anomalies develop in different variants. It was postulated by Burck $\mathrm{HC}$, et al that high origin of the ostia may reduce diastolic blood flow to the coronary arteries $;^{8}$ however, this has been disproved, and in fact, most variations have no clinical significance. Clinically, the most important coronary artery anomaly is the left main coronary artery originating from the right sinus of Valsalva or from the right coronary artery (RCA). This specific anomaly is called anomalous aortic origin of coronary artery (AAOCA). The importance lies in the course of the artery. The left main coronary artery in this situation may take one of four pathways to the left ventricle as illustrated in Figure 2, septal anomalous origins of the left main coronary artery

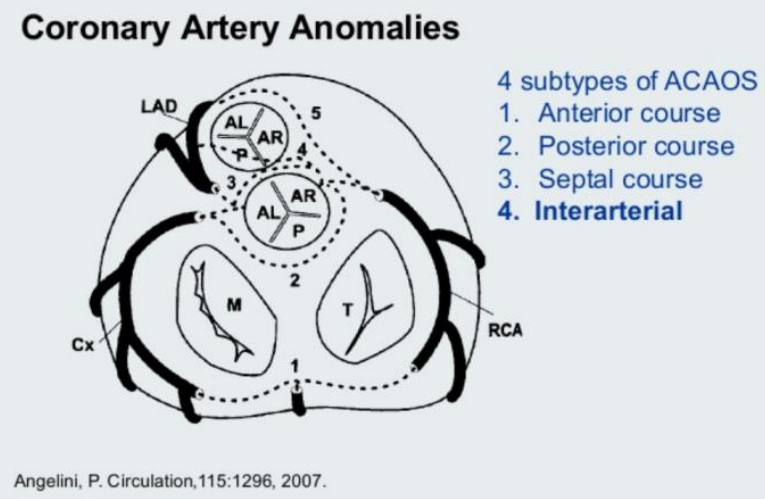

Figure 2. Diagram showing the 4 subtypes of left main coronary artery origin anomalies. Reproduced with permission from Walters Kluwer Health, Inc. (ALMCA), anterior ALMCA, retroaortic ALMCA, and interarterial ALMCA. ${ }^{3}$ 
These four different pathways can be identified during coronary angiography using the "dot and eye method". ${ }^{3}$ In the septal ALMCA, the left main coronary artery takes inter muscular course through the septum and then divides in the mid septum into LAD artery and left circumflex artery forming an ellipse or an eye form shape with the left main coronary artery forming the inferior portion of the eye shape and the circumflex artery forming the superior portion. In the anterior ALMCA pathway, the left main coronary artery runs on the anterior free wall of the right ventricle and then divides at the mid-septum into the LAD and circumflex arteries forming an eye shape with the left main coronary artery forming the superior portion of the eye and the LCX forming the inferior portion. In the retroaortic ALMCA, the left main coronary artery runs posteriorly around the aortic root to the anterior surface of the heart and then divides into LAD and circumflex arteries; during coronary angiography, the retroaortic ALMCA is seen as a dot behind the aorta "posterior dot". In the interarterial ALMCA, the left main coronary artery runs between the aorta and pulmonary artery to the anterior surface of the heart and then divides into the LAD and circumflex arteries; during coronary angiography, the interarterial ALMCA is seen as a dot anterior to the aorta "anterior dot".

The interarterial ALMCA has the most clinical significant in clinical practice because it may result in direct compression on the coronary artery with subsequent acute myocardial infarction (MI) or even sudden death. Alternatively, the left main coronary artery may run intramurally with an acute angle of takeoff which may predispose to blood flow obstruction and subsequent ischemia. ${ }^{9}$ Exercise leads to expansion of the aortic root and the pulmonary arteries which might increase the pressure on the coronary artery. Exercise could also increase the pre-existing angulation of the coronary artery takeoff which explains the increased incidence of sudden death among these patients.

Origin of the RCA from the left sinus of Valsalva or LAD can potentially lead to myocardial ischemia and sudden death though not as commonly as left main coronary. ${ }^{10}$ Anomalous origin of left circumflex (LCX) artery from the RCA has not been reported to have any clinical significance, likely due to posterior course to the left ventricle, but vascular compression can still occur. Coronary arteries may also originate from the pulmonary artery; in this situation, the coronary artery will carry blood with a low oxygen saturation, which reflects the pulmonary artery source. The LAD, LCX artery, and RCA can all originate from the pulmonary artery. RCA originating from the pulmonary artery has less clinical significance when compared to LAD and LCX, but clinical sequela still occurs. ${ }^{11}$ Bland-White-Garland syndrome is an anomalous origin syndrome of LCA from the pulmonary artery. It is the most common type of coronary artery anomaly from the pulmonary artery. ${ }^{12}$

\section{Clinical Presentation of the anomalous origin of the coronary arteries}

The clinical presentation varies greatly based on different types of origin anomalies. Patients are generally asymptomatic, but they may present with chest pain, syncopal episodes, or sometimes with sudden death especially in young patients. ${ }^{6,13}$ The incidence of sudden death from AAOCA of the left coronary artery is higher than the right coronary artery, but the overall incidence is still low. ${ }^{7}$ The clinical presentation of coronary artery anomalies originating from the pulmonary artery depends on the coronary artery involved, myocardial distribution of the coronary artery, the number of the collateral vessels, and the pulmonary vascular resistance. In patients without collateral blood supply, myocardial ischemia with subsequent heart failure occurs early in 
infancy. Patients with sufficient collateral blood supply generally have delayed ischemia and heart failure symptoms until adulthood.

Physical examination is generally unremarkable, but in adult patient with symptoms of heart failure could be remarkable for gallop or holosystolic murmur due to papillary muscle dysfunction secondary to the ischemia. A continuous murmur may be heard due to the flow of blood from the aorta to the pulmonary circulation.

\section{Diagnosis of the anomalous origin of the coronary arteries}

Coronary angiography remains the gold standard in diagnosis of coronary artery anomalies. However, noninvasive techniques such as echocardiogram may be able to establish the diagnosis. ${ }^{14}$ In case of coronary artery anomalous origin from the pulmonary artery, chest X ray may show cardiomegaly. EKG findings in patients with left coronary artery originating from the pulmonary artery might show evidence of ischemia, ST segment changes in the anterolateral leads, or Q waves in leads I, AVL, V5, and V6. ${ }^{14}$ Echocardiogram typically will show a dilated heart with global myocardial dysfunction in patients with coronary arteries anomalies from pulmonary arteries complicated by heart failure. Coronary magnetic resonance angiography (CMRA) is increasingly used to define anomalous coronary anatomy. Coronary computed tomography angiography (CCTA) can also be used to diagnose coronary artery anomalies. The diagnostic accuracy of CMRA and CCTA is almost equal as was demonstrated in a clinical study. ${ }^{15}$ Stress myocardial perfusion imaging (MPI) and stress echocardiogram may also help with the diagnosis of anomalous origin of the coronary arteries. Ultimately, coronary angiography is still the gold standard, and is recommended when other non-invasive studies are not diagnostic and the suspicion of coronary artery anomalies is high. ${ }^{16}$

\section{Association between anomalous origin of the coronary arteries and other congenital heart diseases}

Anomalous origin of the coronary arteries can be associated with some congenital heart diseases. It has been reported that coronary artery anomalies occur in about $9 \%$ of patients with tetralogy of fallot, usually with the LAD arising from the RCA or from the right sinus of Valsalva. ${ }^{17,18}$ Coronary artery anomalies occur in higher frequency in transposition of the great arteries. In 60 $\%$ of patients with transposition of great arteries, the RCA originates from the posterior aspect of the right-facing sinus of Valsalva and left main coronary artery originates from the posterior aspect of the left-facing sinus. ${ }^{19,20}$ In $20 \%$ of patients with transposition of great arteries, the LCX artery arises from the RCA. In patients with truncus arteriosus, a single coronary artery, or displacement of the coronary ostia, has been reported. ${ }^{21}$

\section{Management of anomalous origin of the coronary arteries}

Treatment depends on different types of coronary artery anomalies. If the coronary artery arises from the aorta and is asymptomatic with no evidence of ischemia, surgical intervention is not indicated in most of the cases. Patients with AAOCA, especially with left coronary artery origin from the right coronary cusp, might develop significant myocardial ischemia and surgical intervention is needed in this situation. Coronary artery bypass grafting (CABG) or unroofing (marsupialization) of the coronary artery is a surgical technique used to prevent coronary artery 
compression and subsequent ischemia. ${ }^{22}$ Patients who are not candidates for CABG might benefit from stent placement to prevent vessel compression. ${ }^{23}$ Surgical intervention with reimplantation of the coronary artery to the aorta or tunneling of the coronary artery to the aorta (Takeuchi procedure) is necessary for all patient with coronary artery anomalous origin from the pulmonary artery.

\section{Conclusion}

Anomalous origin of the coronary arteries is a rare finding in clinical practice. Patients are usually asymptomatic but can present with ominous signs based on the specific anomaly they have. Symptomatic patients with AAOCA with left main coronary artery from the right cusp need surgical correction of the anomaly to prevent the catastrophic complication such as MI or sudden death. Coronary artery anomalous origin from the pulmonary artery needs surgical correction of the anomaly to prevent development of heart failure. Stent placement to prevent coronary artery compression is also an interventional option for patients who cannot undergo surgical intervention. It is important that physicians be aware of the different types of anomalous origin of the coronary arteries in order to deliver the best possible care to their patients. 


\section{References}

1. Angelini P. Coronary artery anomalies: an entity in search of an identity. Circulation. 2007;115(10):1296305.

2. Krasuski RA, Magyar D, Hart S, Kalahasti V, Lorber R, Hobbs R, et al. Long-term outcome and impact of surgery on adults with coronary arteries originating from the opposite coronary cusp. Circulation. 2011;123(2):154-62.

3. Serota H, Barth CW, 3rd, Seuc CA, Vandormael M, Aguirre F, Kern MJ. Rapid identification of the course of anomalous coronary arteries in adults: the "dot and eye" method. Am J Cardiol. 1990;65(13):891-8.

4. Brothers J, Gaynor JW, Paridon S, Lorber R, Jacobs M. Anomalous aortic origin of a coronary artery with an interarterial course: understanding current management strategies in children and young adults. Pediatr Cardiol. 2009;30(7):911-21.

5. Yamanaka O, Hobbs RE. Coronary artery anomalies in 126,595 patients undergoing coronary arteriography. Cathet Cardiovasc Diagn. 1990;21(1):28-40.

6. Maron BJ, Carney KP, Lever HM, Lewis JF, Barac I, Casey SA, et al. Relationship of race to sudden cardiac death in competitive athletes with hypertrophic cardiomyopathy. J Am Coll Cardiol. 2003;41(6):974-80.

7. Gersony WM. Management of anomalous coronary artery from the contralateral coronary sinus. J Am Coll Cardiol. 2007;50(21):2083-4.

8. Burck HC. [High and Funnel-Like Origin of the Coronary Arteries]. Beitr Pathol Anat. 1963;128:139-56.

9. Lorenz EC, Mookadam F, Mookadam M, Moustafa S, Zehr KJ. A systematic overview of anomalous coronary anatomy and an examination of the association with sudden cardiac death. Rev Cardiovasc Med. 2006;7(4):205-13.

10. Taylor AJ, Rogan KM, Virmani R. Sudden cardiac death associated with isolated congenital coronary artery anomalies. J Am Coll Cardiol. 1992;20(3):640-7.

11. Lerberg DB, Ogden JA, Zuberbuhler JR, Bahnson HT. Anomalous origin of the right coronary artery from the pulmonary artery. Ann Thorac Surg. 1979;27(1):87-94.

12. Liebman J, Hellerstein HK, Ankeney JL, Tucker A. The Problem of the Anomalous Left Coronary Artery Arising from the Pulmonary Artery in Older Children. Report of Three Cases. N Engl J Med. 1963;269:486-94.

13. Eckart RE, Scoville SL, Campbell CL, Shry EA, Stajduhar KC, Potter RN, et al. Sudden death in young adults: a 25-year review of autopsies in military recruits. Ann Intern Med. 2004;141(11):829-34.

14. Chaitman BR, Lesperance J, Saltiel J, Bourassa MG. Clinical, angiographic, and hemodynamic findings in patients with anomalous origin of the coronary arteries. Circulation. 1976;53(1):122-31.

15. Post JC, van Rossum AC, Bronzwaer JG, de Cock CC, Hofman MB, Valk J, et al. Magnetic resonance angiography of anomalous coronary arteries. A new gold standard for delineating the proximal course? Circulation. 1995;92(11):3163-71.

16. Frommelt PC, Frommelt MA, Tweddell JS, Jaquiss RD. Prospective echocardiographic diagnosis and surgical repair of anomalous origin of a coronary artery from the opposite sinus with an interarterial course. J Am Coll Cardiol. 2003;42(1):148-54.

17. Meng CC, Eckner FA, Lev M. Coronary Artery Distribution in Tetralogy of Fallot. Arch Surg. 1965;90:363-6.

18. Dabizzi RP, Caprioli G, Aiazzi L, Castelli C, Baldrighi G, Parenzan L, et al. Distribution and anomalies of coronary arteries in tetralogy of fallot. Circulation. 1980;61(1):95-102.

19. Wernovsky G, Sanders SP. Coronary artery anatomy and transposition of the great arteries. Coron Artery Dis. 1993;4(2):148-57.

20. Shaher RM, Puddu GC. Coronary arterial anatomy in complete transposition of the great vessels. Am J Cardiol. 1966;17(3):355-61.

21. Shrivastava S, Edwards JE. Coronary arterial origin in persistent truncus arteriosus. Circulation. 1977;55(3):551-4.

22. Romp RL, Herlong JR, Landolfo CK, Sanders SP, Miller CE, Ungerleider RM, et al. Outcome of unroofing procedure for repair of anomalous aortic origin of left or right coronary artery. Ann Thorac Surg. 2003;76(2):589-95; discussion 95-6.

23. Doorey AJ, Pasquale MJ, Lally JF, Mintz GS, Marshall E, Ramos DA. Six-month success of intracoronary stenting for anomalous coronary arteries associated with myocardial ischemia. Am J Cardiol.

2000;86(5):580-2, A10. 